\title{
Room-temperature solid-state maser
}

\section{Mark Oxborrow, Jonathan D. Breeze, and Neil McN. Alford}

Since its discovery, the laser has resulted in many innovations and become a ubiquitous technology. The maser (microwave amplification by stimulated emission of radiation), on the other hand, whilst instrumental to the laser's birth ${ }^{1,2}$, has not. The maser's relative obscurity to date has largely been due to the inconvenience of the operating conditions needed for its various realizations. Atomic ${ }^{3}$ and free-electron ${ }^{4}$ masers require vacuum chambers and pumping. Solid-state masers ${ }^{5}$, though excelling as low-noise amplifiers ${ }^{6}$ and occasionally incorporated within ultra-stable oscillators ${ }^{7,8}$, have required cryogenic refrigeration. Most realizations have also required strong magnets and/or magnetic shielding. Overcoming these obstacles would pave the way for significant new innovations such as more sensitive chemical assays, more precise determinations of biomolecular structure and function, and more accurate medical diagnostics (incl. tomography) based on enhanced magnetic resonance spectrometers ${ }^{9}$ incorporating maser amplifiers and oscillators. Here we report the first experimental demonstration of a solid-state maser operating at room temperature in pulsed mode. It works on a lab bench, in air, in the earth's magnetic field and amplifies at around 1.45 GHz. In contrast to the cryogenic ruby maser ${ }^{6}$, its gain medium is an organic mixed molecular crystal, namely p-terphenyl doped with pentacene, where the latter species is photo-excited by yellow light. The maser's pumping mechanism exploits spin-selective molecular intersystem crossing (ISC) ${ }^{10}$ into pentacene's triplet ground state ${ }^{11,12}$. When configured as an oscillator, the solid-state maser's measured output power of around $\mathbf{- 1 0} \mathbf{~ d B m}$ is approximately 100 million times greater than that of an atomic hydrogen maser ${ }^{3}$, which oscillates nearby in frequency ( 1.42 GHz). By exploiting the high levels of spin 
polarization readily generated via ISC in photo-excited pentacene and other aromatic molecules, this new type of maser appears capable of amplifying with a residual noise temperature far below room temperature.

To the best of our knowledge, no-one has previously attempted to make a zero-field maser ${ }^{13}$ out of an optically pumped crystal of pentacene-doped $p$-terphenyl. Two further novel features of the experiment we report here are (a) a high-Q microwave cavity containing a dielectric ring of sapphire, and (b) a medical laser, designed for the treatment of vascular skin lesions, that is here used to deliver intense pulses of pump light into the pentacene:p-terphenyl maser crystal at its optimal absorption wavelength $(\sim 585 \mathrm{~nm})$.

As key components within a myriad of measurement and telecommunication devices/systems, low-noise amplifiers (LNAs) have been intensively researched towards improving their performance and reducing their operating requirements ${ }^{14}$. As LNAs, conventional solid-state masers ${ }^{5}$ offer low residual noise temperatures ${ }^{6}$ and, in contrast to micro-fabricated devices, are extremely rugged with respect to electromagnetic shock and overloading. Furthermore, they exhibit low intermodulation distortion ${ }^{6}$, so reducing interference from out-of-band signals, and low $1 / f$ noise ${ }^{7}$. The active material within a conventional solid-state maser is a dielectric crystal containing paramagnetic ions, e.g. ruby, that is maintained typically below $10 \mathrm{~K}$ and exposed (with exceptions ${ }^{8,13}$ ) to a strong d.c. magnetic bias field. Despite the inconvenience of these operating conditions, as well as competition from amplifiers based on cooled high-electron-mobility transistors (HEMTs) ${ }^{15}$, ruby masers retain a niche in deepspace communication ${ }^{6}$. They were used, for example, to bring back spectacular images of the solar system's outer planets and their moons from NASA's two Voyager space probes ${ }^{16}$. 
The lowest-noise semiconductor-based amplifiers at microwave frequencies, which today most often incorporate cooled HEMTs ${ }^{15}$, suffer from a particular limitation. On cooling, the HEMT amplifier's residual noise temperature, though low enough for many applications, sticks stubbornly at a few kelvin ${ }^{17}$. Though this "HEMT plateau" can be lowered somewhat by employing lower device powers ${ }^{18}$, it has motivated the successful development of resonant SQUID-based devices ${ }^{17}$ offering substantially lower noise temperatures, albeit over more limited bandwidths. But with both HEMTs or SQUIDs, the attainment of millikelvin noise temperatures demands sub-kelvin refrigeration, as supplied by a dilution refrigerator or its equivalent.

Why conventional solid-state masers ${ }^{5,6}$ cannot be made to work at room temperature is understood $^{19}$. The most serious problem is that the rate of spin-lattice relaxation, $1 / T_{1}$, thus the microwave power required to saturate the maser's pump transition and hence the required thermal cooling power of the maser's refrigerator, increases extremely rapidly with the absolute (lattice) temperature $T$ of the maser crystal. For two-phonon (Raman) scattering off Kramers-type paramagnetic ions in a 3-D lattice well below its Debye temperature, this relaxation rate scales as $T^{9}$. A second problem is that, even if the maser's pump transition(s) can be saturated by pumping hard, the population inversion, hence the maser crystal's gain, scales as $I \times h f_{\text {mas. }} / k T$, where $I \equiv f_{\text {pump }} / 2 f_{\text {mas. }}-1$ is the inversion ratio ${ }^{5}, f_{\text {mas. }}$ and $f_{\text {pump }}$ are the maser signal and pump frequencies, and $h$ and $k$ are Boltzmann's and Planck's constants, respectively. The residual noise temperature of a conventionally pumped solid-state maser amplifier thus scales as $T$, so nullifying the maser's low-noise advantage at higher operating temperatures. It was realized very early on that, at the expense of energy (quantum) efficiency, larger population inversions, thus lower noise temperatures and somewhat higher sustainable operating temperatures, can be obtained, for a fixed $f_{\text {mas. }}$, by increasing $f_{\text {pump }}$, in 
the extreme case by driving optical pump transitions with (laser) light ${ }^{20}$. But optical pumping alone does not obviate the first problem of the rate of spin-lattice relaxation escalating aggressively with temperature ${ }^{21}$.

Blank et al $^{19}$ considered the realization of optically-pumped masers at room temperature using optically excited organic molecules in strong d.c. magnetic fields, focussing largely on $\mathrm{C}_{60}$ and porphyrins known to exhibit long spin-polarization lifetimes $\left(T_{1}\right)$. They judged ${ }^{22}$ the socalled triplet mechanism (TM), of which Figure 1 in this letter is an example, as unsuitable for maser operation at room temperature. By exploiting the more complex, indirect, radicaltriplet-pair mechanism (the triplet state in question being that of etioporphyrin), they got tantalizingly close to maser threshold; see Figure 3 of reference 23 . More recently, the potential of various TM photo-chemistries, including the fullerene derivative PCBM in polystyrene, was assessed towards realizing particle acceleration by stimulated emission of radiation (PASER) at X-band microwave frequencies ${ }^{24}$.

The maser reported here (see Figures 1 and 2) is TM-based. It exploits spin-selective intersystem crossing (ISC) ${ }^{10}$ into the triplet ground state of pentacene molecules doping crystalline $p$-terphenyl ${ }^{11}$, as has been studied in connection with realizations of both dynamic nuclear polarization ${ }^{25}$ and electron spin resonance of single molecules ${ }^{12}$. Upon $\mathrm{S}_{0} \rightarrow \mathrm{S}_{1}$ photoexcitation with yellow pump light (see Figure 1), ISC provides a strongly inverted spin population between the uppermost $(\mathrm{X})$ and lowest $(\mathrm{Z})$ spin sub-levels of pentacene's triplet ground state ${ }^{11,12}$. The relatively high zero-field splitting (as compared with PCBM, for example) makes it practicable to use a microwave resonator with no applied d.c. magnetic field, so greatly simplifying the experiment setup. Our chosen design of resonator (Figure 2) supports a frequency-tuneable $\mathrm{TE}_{01 \delta}$ mode with a mode volume $\approx 50 \mathrm{~cm}^{2}$. With the pentacene: $p$-terphenyl crystal in place, this mode's unloaded quality factor $(Q)$ was measured 
to be around $2 \times 10^{5}$. When critically coupled through a magnetic loop probe in the side of the resonator's enclosing metal can (see Figure 2), it offered a field-to-power conversion factor (proportional to the square root of the mode's magnetic Purcell factor ${ }^{26}$ ) $\approx 5$ gauss $/ \sqrt{ } \mathrm{W}$.

An unpolarized beam, $\sim 10 \mathrm{~mm}$ in diameter, of yellow $(585 \pm 5 \mathrm{~nm})$ pump light from a Candela SPTL-1b long-pulsed rhodamine-6G dye laser was directed at the maser crystal. This laser was capable of delivering up to $4 \mathrm{~J}$ in a single pulse, at a rate of one pulse per second, though pulses of only $0.5 \mathrm{~J}$ (the time-integrated power of the yellow trace in Figure 3 -as confirmed with a Molectron JMAX 43 pyroelectric joulemeter) were used in our experiment. We estimate that $\sim 0.3 \mathrm{~J}$ was absorbed in the maser crystal per pulse. With the frequency of the $\mathrm{TE}_{01 \delta}$ mode set near to the centre of the $\mathrm{X}-\mathrm{Z}$ spin transition, each pump pulse absorbed by the crystal produced a vigorous burst of maser oscillation - see Figure 3 . The oscillation's magnitude as a function of the $\mathrm{TE}_{01 \delta}$ mode's frequency is shown in Figure 4. The instantaneous output power of the maser burst (blue trace in Figure 3), as monitored directly by a microwave receiver in the form of an HP8593E spectrum analyser set to fixed-frequency mode, was observed to exceed $-10 \mathrm{dBm}$. Over 1000 pump pulses, no change in the size or shape of the maser burst was noticeable provided the resonator was kept in tune. By inserting neutral density filters into the pump beam so as to snub out the maser oscillation, the threshold pump energy exiting from the laser was determined to be $80 \mathrm{~mJ}$ over an effective pump pulse duration of $350 \mu \mathrm{s}$, corresponding to a threshold pump power of about $230 \mathrm{~W}$. Just above threshold, a considerably diminished maser burst, delayed in time and somewhat shorter in duration, could be observed.

We have constructed a simple model for how both the spin populations in the $\mathrm{X}$ and $\mathrm{Z}$ sublevels and the energization of (i.e. photon number in) the maser mode change as functions of time in response to the applied optical pump pulse; see Methods (online) for details. The 
maser output power predicted by this model was fitted to the experimentally observed maser burst, yielding the green curve in Figure 3, together with estimates of three spin-dynamics parameters. Our modelling suggests that near-zero-field operation is particularly advantageous for masing: the rate of spin-lattice relaxation is low and the transition linewidth is narrow ${ }^{27}$.

Our experimental maser oscillator, as shown in Figure 2, can be reconfigured as a pulsedmode amplifier of weak electromagnetic signals by connecting its coupling loop to one port of a circulator ${ }^{5}$. A detailed, quantitative analysis of this amplifier's noise performance, taking into account deleterious sources of noise associated with losses within this circulator and the maser cavity itself, is provided in this letter's Methods (online). The red curve in Figure 3 plots the population ratio $r \equiv N_{\mathrm{X}} / N_{\mathrm{Z}}$ between the upper (X) and lower (Z) maser levels as predicted by our fitted model in response to the optical pump pulse shown (yellow trace). In contrast to saturation-limited maser oscillation (the blue and green curves in Figure 3), this red curve assumes that the maser is operating in the low-signal regime where the energization of the maser mode is miniscule. Though decaying with time, $r$ remains in excess of 2 for up to $150 \mu \mathrm{s}$ from the onset of the pump pulse, implying that the intrinsic limit on the maser amplifier's residual noise temperature stays below $140 \mathrm{mK}$ out to this same time. Whether or not the population ratio $r$ can be sustained sufficiently above unity to allow continuous maser operation (given a suitable pump source and adequate cooling) would appear to be finely balanced with respect to the values of relevant relaxation parameters.

When down-converted, sampled and Fourier-transformed, each burst of maser oscillation exhibited a linewidth of $\sim 6 \mathrm{kHz}$, consistent with that of a perfect sinewave modulated by the maser burst's envelope (blue/green curves in Figure 3). Precise measurements of the maser's noise await continuous operation. 
In conclusion, zero-field maser operation at room temperature with a solid-state gain medium has been demonstrated. By improving the quality of the maser crystal, the effectiveness of the optical pumping, and the Purcell factor of the resonator, we believe that the maser's threshold pump power can be substantially reduced, its efficiency (hence output power as an oscillator) further increased, and its residual noise temperature as an amplifier reduced. The application of a d.c. magnetic field bias should allow the maser's operating frequency to be tuned somewhat. The prospect of continuous maser operation, though not resolved here, remains most tantalizing and motivates attempts to identify different (yet perhaps structurally related) molecular systems, whose properties are yet more advantageous for masing than those of pentacene:p-terphenyl.

Extremely low-noise amplifiers enable the detection of weak yet potentially important electromagnetic signals, which would otherwise be lost. Low-phase-noise oscillators are essential components within frequency standards and clocks. Ultra-sensitive parametric probes and detectors can, moreover, be derived from such oscillators. By being capable of operating at room temperature and in weak ambient magnetic fields (such as the earth's), the maser reported here thus opens up new routes to the making of measurements at unprecedented levels of sensitivity and precision in areas such as space exploration, molecular biology, and quantum computing -to name but three. Furthermore, the general exploitation of intersystem crossing as an effective source of spin-polarized "fuel" for the powering of more intricately orchestrated quantum-molecular processes (beyond merely that of masing through a common microwave field) would appear to be a most fertile area of study. 


\section{Methods summary}

The maser crystal was grown using the vertical Bridgman-Stockbarger method in a doublewalled glass vial ${ }^{28}$. Its constituent $p$-terphenyl was moderately zone refined prior to growth; the substitutional concentration of its pentacene dopant was approximately 0.01 percent. The as-grown crystal boule was trimmed then polished. It exhibited extensive twinning (as revealed by spatially inhomogeneous birefringence) and other internal flaws (see Figure 2).

The microwave resonator was designed using both mode-matching and finite-elementmethod $^{29}$ software. The optical (c) axis of its monocrystalline sapphire ring lay parallel to the ring's geometric axis of rotation to within a tolerance of \pm 1 degree. All of the ring's surfaces and the internal surfaces of the resonator's silver-plated metal can were polished. Upon inserting the pentacene:p-terphenyl boule into the ring, the $\mathrm{X}-\mathrm{Z}$ transition was initially located by directly monitoring on an oscilloscope the response of an L-band Robinson oscillator bridge (which incorporated the resonator within its microwave circuit) to pulses of belowthreshold yellow pump light.

The spin populations in the triplet ground state's $\mathrm{X}$ and $\mathrm{Z}$ sub-levels, together with the photon-number in the $1.45 \mathrm{GHz} \mathrm{TE}_{01 \delta}$ microwave mode, were modelled by coupled linear rate equations capturing the processes of stimulated emission/absorption, spin-lattice relaxation, and ISC-mediated population into, and decay out of, the triplet sub-levels ${ }^{30}$; see Methods (online) for further details including estimates of parameters that enter these equations as coefficients.

The predicted noise performance of our maser is based on formulae derived in Chapter 8 (entitled "Noise -in masers and in general") of Siegman's classic treatise ${ }^{5}$, as have been applied without low-temperature approximations to cryogenic ruby masers by Clauss and 
Shell ${ }^{6}$. Noise contributions associated with losses in passive microwave components (including the resonator), as well as the "intrinsic" maser noise associated with the effective spin temperature across the $\mathrm{X}-\mathrm{Z}$ transition, are all included. 


\section{References}

1. Schawlow, A. L. \& Townes, C. H. Infrared and optical masers, Phys. Rev. 112, 19401949 (1958).

2. Maiman, T. H. Stimulated optical radiation in ruby. Nature 187, 493-494 (1960).

3. Kleppner, D., Goldenberg, H. M. \& Ramsey, N. F. Properties of the hydrogen maser. Appl. Opt. 1, 55-60 (1962).

4. Konoplev, I. V. et al. Experimental study of coaxial free-electron maser based on twodimensional distributed feedback. Phys. Rev. Lett. 96, 035002 (2006).

5. Siegman, A. E. Microwave Solid-state Masers (McGraw-Hill, 1964).

6. Clauss, R. C. \& Shell, J. S. Ruby masers. Chapter 3 of Low-Noise Systems in the Deep Space Network, ed. Reid, M. S., Deep Space Communication and Navigation Series (JPL, Caltech, 2008).

7. Dick, G. J. \& Wang, R. T. Ultra-stable performance of the superconducting cavity maser. IEEE Trans. Instrum. Meas. 40, 174-177 (1991).

8. Bourgeois, P.-Y. et al. Maser oscillation in a whispering-gallery-mode microwave resonator. Appl. Phys. Lett. 87, 224104 (2005).

9. Mollier, J. C., Hardin, J. \& Uebersfeld, J. Theoretical and experimental sensitivities of ESR spectrometers using maser techniques. Rev. Sci. Instrum. 44, 1763-1771 (1973).

10. van der Waals, J. H. EPR of photo-excited triplet states: a personal account. Appl. Magn. Reson. 20, 545-561 (2001). 
11. Sloop, D. J., Yu, H.-L., Lin, T.-S. \& Weissman, S. I. Electron spin echoes of a photoexcited triplet: pentacene in p-terphenyl crystals. J. Chem. Phys. 75, 3746-3757 (1981).

12. Köhler, J., Brouwer, A.C.J., Groenen, E.J.J., Schmidt, J. On the intersystem crossing of pentacene in p-terphenyl, Chem. Phys. Lett. 250 137-144 (1996).

13. Bogle, G. S. \& Symmons, H. F. Zero-field masers. Aust. J. Phys. 12, 1-20 (1959).

14. Whelehan, J. J. Low-noise amplifiers -then and now. IEEE Trans. Microwave Theory Tech. 50, 806-813 (2002).

15. Wadefalk, N. et al. Cryogenic wide-band ultra-low-noise IF amplifiers operating at ultra-low DC power. IEEE Trans. Microwave Theory and Tech. 51, 1705-1711 (2003).

16. Smith, B. A. et al. A new look at the Saturn system: the Voyager 2 images. Science 215, 504-537 (1982).

17. Asztalos, S. J. et al. SQUID-based microwave cavity search for dark-matter axions. Phys. Rev. Lett. 104, 041301 (2010).

18. Korolev, A. M., Shnyrkov, V. I. \& Shulga, V. M. Note: ultra-high frequency ultra-low dc power consumption HEMT amplifier for quantum measurements in millikelvin temperature range. Rev. Sci. Instrum. 82, 016101 (2011).

19. Blank, A., Kastner, R. \& Levanon, H. Exploring new active materials for low-noise room-temperature microwave amplifiers and other devices. IEEE Trans. Microwave Theory Tech. 46, 2137-2144 (1998).

20. Hsu, H. Optical pumping of microwave masers. Proc. IEEE, January 1963, 185-189. 
21. Sabisky, E. S. \& Anderson, C. H. A solid-state cw optically pumped microwave maser. Appl. Phys. Lett. 8, 298-300 (1966).

22. Blank, A. \& Levanon, H. Toward maser action at room temperature by triplet-radical interaction and its application to microwave technology. RIKEN Review 44, February 2002, 128-130.

23. Blank, A. \& Levanon, H. Application of photo-induced electron spin polarization at room temperature to microwave technology. Appl. Phys. Lett. 79, 1694-1696 (2001).

24. Antipov, S. et al. Microwave active media studies for paser. Proceedings of PAC09, FR5RFP001, 1-3 (Vancouver, BC, Canada, 2009).

25. Kagawa, A., Murokawa, Y.,Takeda, K. \& Kitagawa, M. Optimization of ${ }^{1} \mathrm{H}$ spin density for dynamic nuclear polarization using photo-excited triplet electron spins. J. Magn. Reson. 197, 9-13 (2009).

26. Purcell, E.M. Spontaneous Emission Probabilities at Radio Frequencies. Phys. Rev. 69, 681-686 (1946).

27. Lang, J., Sloop, D. J. \& Lin, T.-S. Orientational anisotropic studies by field rotation technique: Near zero-field pulsed EPR experiments of pentacene in $p$-terphenyl. Journal of Magnetic Resonance 176, 249-256 (2005).

28. Selvakumar, S. et al. Growth and studies on SSVBT grown $p$-terphenyl single crystals. J. Cryst. Growth 275, e265-e271 (2005).

29. Oxborrow, M. Traceable 2-D finite-element simulation of the whispering-gallery modes of axisymmetric electromagnetic resonators. IEEE Trans. Microwave Theory Tech. 55, 1209-1218 (2007). 
30. Takeda, K., Takegoshi, K. \& Terao, T. Zero-field electron spin resonance and theoretical studies of light penetration into single crystals and polycrystalline material doped with molecules photoexcitable to the triplet state via intersystem crossing. J. Chem. Phys. 117, 4940-4946 (2002). 


\section{Acknowledgements}

M.O. thanks John Sherwood (Univ. of Strathclyde) for advice on the growth of molecular crystals by the Bridgman-Stockbarger method, and Robert Ferguson and Conway Langham (both at NPL) for advice on how best to cut and polish the crystalline $p$-terphenyl boule and for CAD-designing the metal components of the microwave resonator, respectively. M.O. and J.D.B. thank Chris Kay (University College London) for discussions and guidance on pulsedEPR spectroscopy. This work was supported by the NMS Pathfinder Metrology Programme and by the Engineering and Physical Sciences Research Council.

\section{Author contributions}

J.D.B. performed initial pulsed-EPR experiments with M.O., and analysed the resultant data. Both J.D.B. and M.O. carried out independent electromagnetic modelling of the $\mathrm{TE}_{01 \delta^{-}}$-mode microwave resonator using different software. M.O. grew the pentacene:p-terphenyl crystal, designed and constructed the microwave circuitry and optical systems, performed the final experiments and analysed the resultant data. N.M.A. initiated the original work on high-Q cavities. N.M.A. and M.O. wrote the paper.

\section{Author information}

Reprints and permissions information is available at www.nature.com/reprints. The authors declare no competing financial interests. Correspondence and requests for materials should

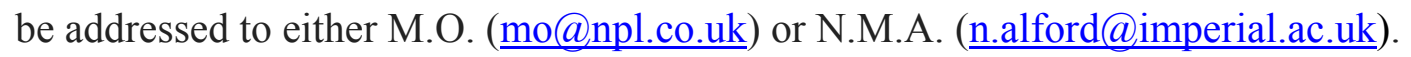




\section{Figure legends}

Figure 1: Maser pumping scheme (Jablonski diagram). Pentacene guest molecules within a $p$-terphenyl host lattice are driven from their singlet ground states $\mathrm{S}_{0}$ into their first-excited single states $\mathrm{S}_{1}$ by absorbing photons of yellow pump light. From $\mathrm{S}_{1}$, they predominantly undergo intersystem crossing (ISC) into their triplet ground states $\mathrm{T}_{1}$. The ISC process is spin selective, where the relative population rates (splitting ratios) ${ }^{11,12}$ into $T_{1}$ 's three spin sublevels are represented by solid black circles. As can be seen, the uppermost (X) sub-level is preferentially populated resulting in a strong initial population inversion between it and the lowest (Z) sub-level, so providing the conditions for masing across the $\mathrm{X}-\mathrm{Z}$ transition.

Figure 2: Anatomy of the maser. A crystal of $p$-terphenyl doped with pentacene is located in the a.c. magnetic field of the $\mathrm{TE}_{01 \delta}$ mode of a microwave resonator and illuminated with a beam of yellow light from a pulsed dye laser. By maser action, the $\mathrm{TE}_{01 \delta}$ mode is energized, from which a signal is extracted by a magnetic coupling loop. The photograph on the right details the resonator's sapphire ring and pentacene:p-terphenyl crystal slotted inside it; yellow back lighting reveals flaws within the crystal.

Figure 3: Maser response in the time domain. The amber trace (scale on near left) is the pump laser's instantaneous output power, integrating to $\sim 0.5 \mathrm{~J}$ per pulse. The blue trace (scale on right) is the instantaneous power of the maser's oscillation burst as monitored with a microwave receiver at a resolution bandwidth of $10 \mathrm{MHz}$ centred on $1.45 \mathrm{GHz}$. The green trace (same scale on right) is our fitted model's prediction of same. The red curve (scale on far left) is the predicted population ratio between the upper $(\mathrm{X})$ and low $(\mathrm{Z})$ maser levels in response to the optical pump in the low-signal regime. 
Figure 4: Frequency response of maser action. The hollow black circles and associated black guide line plot the measured plateau amplitude of the maser oscillation (vertical scale, obtained by down-converting the oscillation's output to a few tens of $\mathrm{kHz}$ and observing the resultant "beat" on a digital storage oscilloscope) upon illuminating the pentacene:p-terphenyl crystal with a $\sim 0.5 \mathrm{~J}$ pulse of yellow light, for different frequencies of the microwave resonator's $\mathrm{TE}_{01 \delta}$ mode (horizontal scale). 


\section{Methods}

Maser crystal. The pentacene was of commercial origin and donated by Sandrine Heutz of Dept. Materials, Imperial College. The $p$-terphenyl was purchased from Alfa Aesar (99+\% grade) and thereupon moderately zone-refined: sealed in glass tubes under dry argon and swept repeatedly at $0.5 \mathrm{~mm} /$ minute up through a vertical furnace. A double-walled ${ }^{28}$ vial of borosilicate glass (ID $18 \mathrm{~mm}$, height $70 \mathrm{~mm}$; made by Cambridge Glassblowing Ltd) was charged with a mixture of pentacene and zone-refined $p$-terphenyl in a stoichiometric ratio of approximately $1: 10^{4}$. Due to oxygen contamination during growth, the actual concentration of pentacene surviving in the crystal may have been somewhat lower than this. The vial was lowered through a vertical tube furnace at a rate of $1 \mathrm{~mm} /$ hour. A clean, pink crystal formed though, upon cooling to room temperature at a rate of approximately $-50^{\circ} \mathrm{C}$ per hour, many flaws appeared within it due to twinning and/or thermal shock. The vial was carefully cracked open and the crystal boule removed intact from it. This boule was then gently cut ("topped and tailed") with a circular saw and then polished, the last abrasive being opaline. The $p$ terphenyl crystal's $a-b$ cleavage plane contained the boule's rotation axis to within \pm 5 degrees. Extensive twinning meant that the crystal's $a$ and $b$ axes were differently oriented in different parts of the crystal (the pump beam always illuminated several such domains).

Microwave resonator. The resonator's basic aspect ratios and dimensions were modelled using in-house-written mode-matching software towards realizing a high-Q mode at 1.45 GHz. A "2.5-D” FEM-based electromagnetic mode simulator ${ }^{29}$ platformed on COMSOL, in conjunction with AutoCAD, was additionally used to determine suitable shapes and dimensions for all components. The polished sapphire ring was sourced from SurfaceNet GmbH, Germany. The metal can (incl. piston) was plated with a 30- $\mu \mathrm{m}$ layer of non-bright silver at Platerite, Aldershot, UK, then polished by hand. 
Modelling of the spin dynamics. We assume that the spin populations in the triplet ground state's $\mathrm{X}$ and $\mathrm{Z}$ sub-levels, and the photon-number in the $1.45 \mathrm{GHz} \mathrm{TE}_{01 \delta}$ microwave mode are governed by coupled linear rate equations, with terms capturing the processes of stimulated emission/absorption, spin-lattice relaxation, and ISC-mediated population into, and decay out of, the triplet sub-levels. [The Y sub-level is not included in the model as this would have introduced several more free parameters.] We ignore both (i) the spatial variation of the $\mathrm{TE}_{01 \delta}$ mode's magnetic field across the pumped region of the pentacene:p-terphenyl crystal and (ii) the vectorial complexities associated with differently oriented pentacene molecules at different $p$-terphenyl lattice sites, in relation to the directions of both the (at least initially unpolarized) pump beam and the $\mathrm{TE}_{01 \delta}$ mode's magnetic field. Elaborations necessary to include the effects of inhomogeneous broadening and spin diffusion are not included; we assume a simple Lorentz-broadened $\mathrm{X}-\mathrm{Z}$ transition. We estimate that, thanks to the large diameter of the pump beam and the low concentration of pentacene within the maser crystal (so increasing its absorption depth to the pump light), the heat generated by a single pump pulse shifts the frequency of the $\mathrm{X}-\mathrm{Z}$ transition by only a small fraction of its linewidth; we thus ignore thermal effects.

We have estimated (based in part on a measurement using an optical power meter placed inside the sapphire ring) that, due to reflections and scattering off poorly polished surfaces, only about $60 \%$ of the pump laser's output gets absorbed by the maser crystal; this optical loss is included in our model. On further assuming that (i) the value for the room-temperature intersystem crossing yield is $62.5 \%$, as deduced by Takeda et al ${ }^{30}$, and (ii) the values of the ISC population ratios $\left(p_{\mathrm{X}}^{\mathrm{ISC}}=0.76, p_{\mathrm{Z}}^{\mathrm{ISC}}=0.08\right)$ and the decay lifetime of the $\mathrm{Z}$ sub-level $\left(\tau_{\mathrm{Z}}=10^{-3} \mathrm{~s}\right)$ are those determined by Sloop et $\mathrm{al}^{11}$ from X-band spin-echo measurements, we estimate that the ratio of the decay lifetimes between the $\mathrm{Z}$ and $\mathrm{X}$ sub-levels $\tau_{\mathrm{Z}} / \tau_{\mathrm{X}} \approx 8.5$, the 
effective linewidth of the $\mathrm{X}-\mathrm{Z}$ transition $\Delta f \equiv 1 /\left(\pi T_{2}\right) \approx 860 \mathrm{kHz}$ and the spin-lattice relaxation time $T_{1} \approx 135 \mu$ s with uncertainties of \pm 20 percent on all three parameters, which were the only free parameters fitted. The green curve appearing in Figure 3 is the model's prediction for the above parameter values. Our estimate of the linewidth agrees with the value of $700 \mathrm{kHz}$ reported by Lang et al. ${ }^{27}$, and is broadly consistent with an estimate for the spin-spin relaxation time of $T_{2} \approx 260 \mathrm{~ns}$ extracted from a preliminary measurement on a small, more concentrated polycrystalline sample of pentacene:p-terphenyl using a standard X-band pulsed EPR spectrometer. Figure 4 would suggest, however, that our imperfect maser crystal suffers additional inhomogeneous broadening of a few MHz. Our estimates of $\tau_{\mathrm{z}} / \tau_{\mathrm{X}}$ and $T_{1}$ are factors of two lower and several higher, respectively, when compared to the values of their high-magnetic-field counterparts, as determined by Sloop et al. ${ }^{11}$. We have yet to study the zero-field $\mathrm{Y}-\mathrm{Z}$ and $\mathrm{X}-\mathrm{Y}$ transitions at $\sim 1.34 \mathrm{GHz}$ and $\sim 107 \mathrm{MHz}$ respectively (the latter requires a different sort of resonator suitable for $\mathrm{VHF}$ ), as would be needed to determine all of the parameters within a complete model of the spin dynamics -including transitions to and from the Y sub-level.

Amplifier residual noise temperature. Configured as a cavity amplifier operated in reflection through a circulator, the maser's residual noise temperature can be formulated ${ }^{5,6}$ as

$$
T_{\text {noise }}=(1+1 / G) \frac{L_{\text {circ. }}^{\mathrm{dB}}}{e^{\mathrm{dB}}} T_{0}+(1-1 / G)\left[\frac{L_{\text {cav. }}^{\mathrm{dB}}}{G^{\mathrm{dB}}} T_{0}+\frac{G_{\text {mas. }}^{\mathrm{dB}}}{G^{\mathrm{dB}}} \frac{r}{(r-1)} \frac{h f_{\text {mas. }}}{k}\right] \text {, }
$$

where $X^{\mathrm{dB}} \equiv 10 \log _{10}[X]$ denotes a quantity's decibel equivalent $\left(e^{\mathrm{dB}} \approx 4.343\right) ; L_{\text {circ. }}^{\mathrm{dB}}$ is the insertion loss through the circulator; $G^{\mathrm{dB}} \equiv G_{\text {mas. }}^{\mathrm{dB}}-L_{\text {cav. }}^{\mathrm{dB}}$ is the amplifier's overall logarithmic power gain, where $G_{\text {mas. }}^{\mathrm{dB}}$ is the gain associated with the masing process, and $L_{\text {cav. }}^{\mathrm{dB}}$ is the passive loss of the "dark" (unpumped) cavity in reflection, $T_{0}$ is the operational temperature of the 
cavity and circulator -taken here to be $293 \mathrm{~K}, f_{\text {mas. }} \approx 1.45 \mathrm{GHz}$, and $r \equiv N_{\mathrm{X}} / N_{\mathrm{Z}}$ is the spin population ratio between the upper $(\mathrm{X})$ and lower $(\mathrm{Z})$ maser levels. We now assume that the amplifier is good: i.e. that its power gain $G \gg 1$, and $G_{\text {mas. }}^{\mathrm{dB}} \gg L_{\text {cav. }}^{\mathrm{dB}}$. By using a low-loss circulator of say $L_{\text {cav. }}^{\mathrm{dB}} \approx 0.1 \mathrm{~dB}$, the first term is (1) can be reduced to $\sim 7 \mathrm{~K}$. The middle term in (1) proportional to $L_{\text {cav. }}^{\mathrm{dB}} / G^{\mathrm{dB}}$ originates from Ohmic and/or dielectric losses in the cavity. When set to its maximum output power, the pump laser used in our experimental maser can provide sufficient light to exceed maser threshold by a factor of approximately 50, i.e. $L_{\text {cav. }}^{\mathrm{dB}} / G^{\mathrm{dB}} \approx 0.02$, giving this middle term a value of around $6 \mathrm{~K}$; this noise contribution could be reduced further by devising a cavity with a higher magnetic Purcell factor or using a laser that supplies pulses of higher instantaneous pump power (though probably of only shorter duration). This leaves the last, "intrinsic" term in (1) proportional to $r /(r-1)$. The advantage of pumping optically via ISC is that $r$ can be considerably greater than unity even at room temperature. Naïvely taking this ratio to be that of the ISC process (Figure 1) alone, i.e. $r=0.76 / 0.08=9.5$, the intrinsic term contributes a noise temperature of just $78 \mathrm{mK}$. In reality, $r$ will be pulled down by relaxation processes as time increases -see the red curve in Figure 3, so raising this noise temperature. But the maser amplifier's overall noise temperature may still be deeply cryogenic for long enough to render the amplifier advantageous in certain pulse-mode applications such as the amplification of radar echoes. Various improvements spring to mind here, like the construction of a travelling-wave maser ${ }^{5,6}$, so obviating the circulator's up-front noise contribution, in a narrow yet low-loss ("slowwave") waveguide to keep $L_{\text {cav. }}^{\mathrm{dB}} / G^{\mathrm{dB}}$ low. 


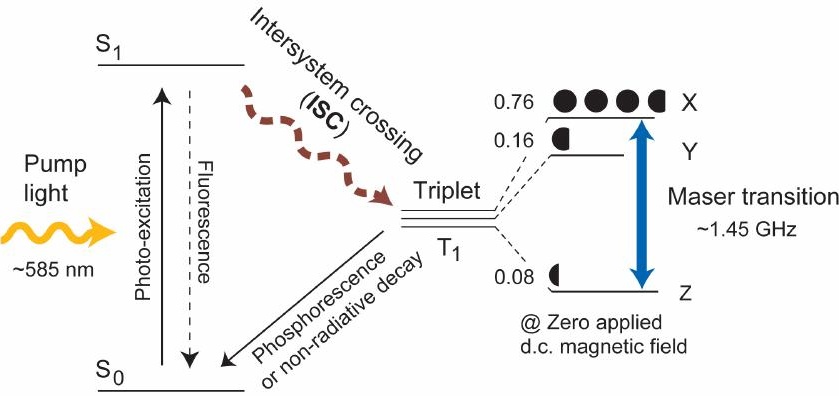




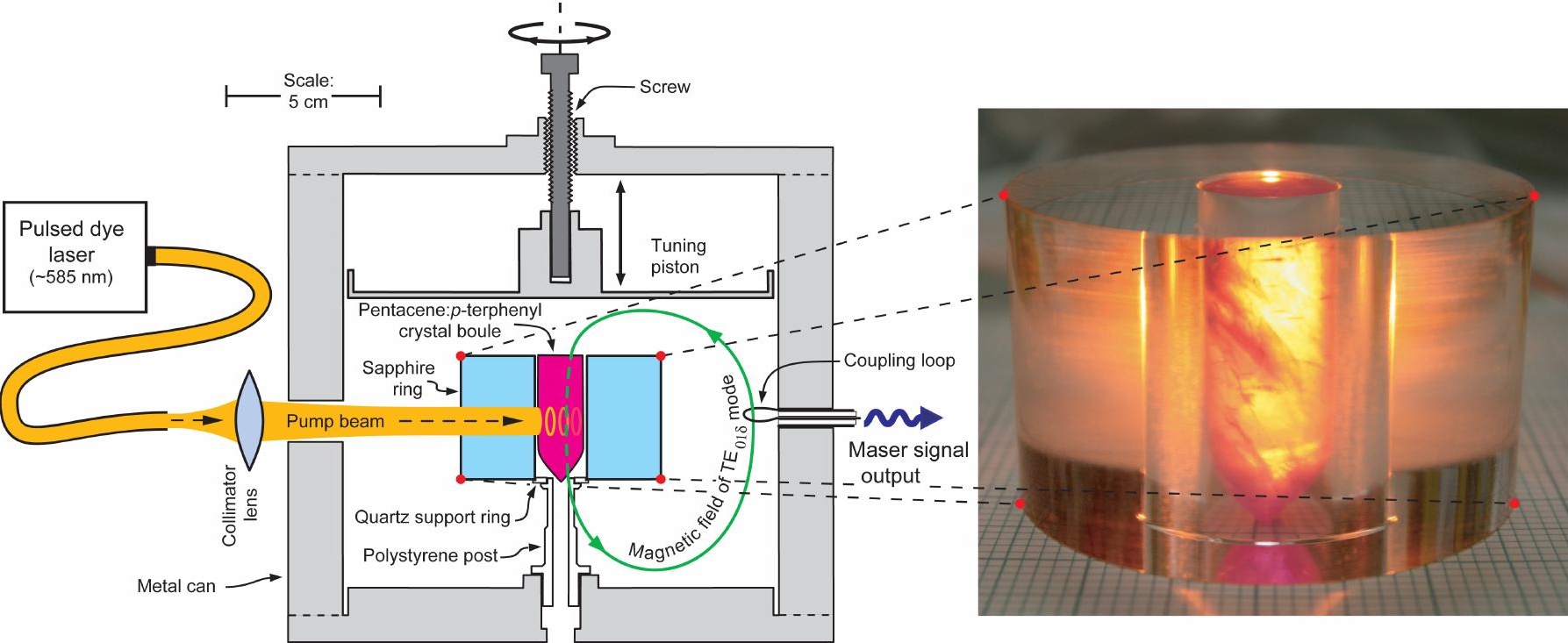




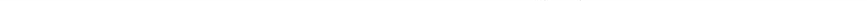




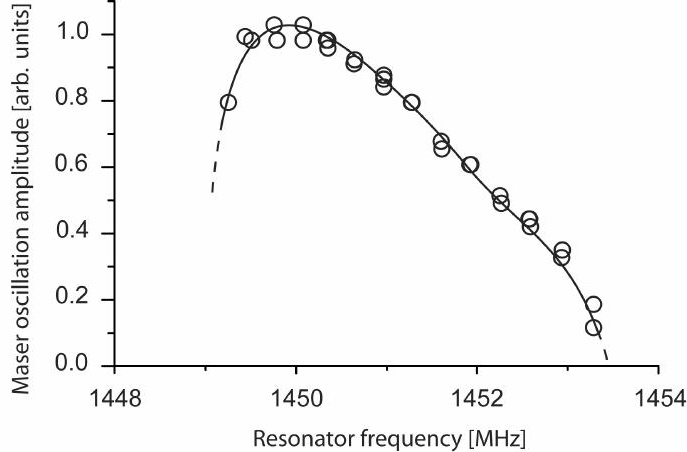

